\title{
STRONG GRAVITY AND X-RAY SPECTROSCOPY
}

\author{
A. MACIOŁEK-NIEDŹWIECKI \\ Łódź University, Department of Physics \\ Pomorska 149/153, 90-236 Eódź, Poland \\ AND \\ P. MAGDZIARZ \\ University of Durham, Department of Physics \\ South Road, Durham DH1 3LE, UK
}

\begin{abstract}
This paper reviews the effects of general relativity in an Xray spectrum reflected from a cold matter accreting onto a black hole. The spectrum consists of the iron $\mathrm{K} \alpha$ line and the Compton reflection. We sketch the overall picture of radiative processes in the central parts of the accretion flow with relation to the relativistic effects derived from the discrete features in the X-ray spectrum. We discuss implications for detection of relativistic effects and computational tools of spectral analysis.
\end{abstract}

\section{Introduction}

Among various observational signatures of accreting black holes (e.g., Madejski 1998; Paradijs 1998) perhaps the most convincing source of information on the strong gravitational field in the vicinity of the black hole comes from $\mathrm{X}$-ray spectroscopy. In the central part of the accreting flow the emitting matter exists in at least two phases: a hot, mildly relativistic, moderately optically thick $(\tau \sim 1)$ plasma emitting hard X-ray continuum (e.g., Poutanen 1998) and a cold, Compton-thick, thermal plasma of temperatures characteristic for optical/UV/soft X-ray energy range (e.g., Blaes 1998). Such two emitting phases of matter are radiatively coupled to each other, leading to a complex variable geometry of the accreting flow (e.g., Życki, Done \& Smith 1998; Magdziarz et al. 1998). However, both the multi-temperature thermal continuum emitted by the cold matter and the hard X-ray continuum produced by Comptonization of the soft photons by the hot, thermal 
plasma are less useful for probing the geometry of the emitting source: the first one, due to complex atomic physics and superposition of emission from mostly more distant components, and the second one due to basic featureless continuum character. On the other hand, illumination of the cold matter by the hard X-rays produces Compton reflection component with superimposed discrete signatures coming from bound-free absorption and fluorescence lines (e.g., George \& Fabian 1991). The most pronounced of these are the iron absorption edge and the iron $\mathrm{K} \alpha$ fluorescence line, at 7.1 $\mathrm{keV}$ and $6.4 \mathrm{keV}$, respectively (for the neutral matter), both discovered in the X-ray spectra of black hole systems by Ginga (e.g., Pounds et al. 1990). Modeling the energy shifts of those spectral features (with respect to the known rest energies) we can investigate physical conditions in the vicinity of the black hole, where the generation of the X-ray luminosity takes place. $A S C A$ observations have confirmed that the X-ray spectra contain a significant contribution from the matter emitting in the immediate vicinity of the black hole, however we need a much higher quality data to investigate the velocity field of that matter, its physical state, and the gravitational potential in the emission region.

In this paper we review the theoretical models developed for the analysis of relativistic effects in the spectra generated by black hole accretion (section 2) and summarize the observational data indicating presence of such effects (section 3). We also discuss the possibility of the estimation of the black hole spin by modelling the X-ray spectral features (section 4).

\section{Physical outline}

In order to investigate the relativistic distortion affecting the spectrum of the radiation coming from the region with the strong gravitational field, in particular to obtain the precise profiles of the discrete features, one has to solve directly the geodesic equation. No analytical solutions are available apart from the Fabian et al. (1989) formulae for the emission line profile from a disk around a Schwarzschild black hole. However, these formulae fail in the strong field limit. Therefore, the calculation of the relativistic effects requires quite complex numerical procedures.

Since the publication of the paper by Bardeen (1970) it became clear that black holes in accretion powered systems should be described by the Kerr metric rather than the less general Schwarzschild metric, as the accretion is likely to spin up the black hole. The Kerr black hole can be characterized by two parameters, the mass, $M$, and the dimensionless spin parameter, $a$. The maximum value of $a=0.998$ cannot be exceeded in accretion systems (Thorne 1974) due to a torque exerted on the hole by radiation from the accreting matter (cf. Moderski \& Sikora 1996 for the 
recent calculation of the black hole spin evolution).

All the relativistic effects affecting the spectra, except the variability time-scale, do not depend on the absolute distance from the black hole, and are functions of the distance in units of the gravitational radius, $r_{\mathrm{g}}=$ $G M / c^{2}$, only. Therefore, the same quantitative effects would occur for both the stellar mass $\left(10 M_{\odot}\right)$ and supermassive $\left(10^{6}-10^{9} M_{\odot}\right)$ black holes. The value of the spin influences the trajectories close to the black hole yielding the distance of the last (quasi) stable circular orbit $r_{\mathrm{ms}}=6 r_{\mathrm{g}}$ for $a=0$ and $r_{\mathrm{ms}}=1.23 r_{\mathrm{g}}$ for $a=0.998$.

The velocity field of the accreting matter is usually treated in the approximated way in models of relativistic effects: it is assumed that the matter accretes in a form of a geometrically thin accretion disk (cf. Jaroszyński \& Kurpiewski 1997 for the observational effects in geometrically thick disks expected in low-efficiency systems) and, for $a>0$, it is assumed that the disk rotates in the planar plane of the black hole (cf. Bardeen \& Petterson 1975 for frame dragging effects on disks not in the equatorial plane). The accreting material is assumed to flow along circular equatorial geodetics with a superimposed small radial inflow (neglected in calculations) for $r>r_{\mathrm{ms}}$, and to be in free fall for $r<r_{\mathrm{ms}}$.

The first calculation of the spectrum of the matter accreting onto a Kerr black hole was presented by Cunningham (1975). His results, however, do not have the form suitable for data analysis, as they are tabulated only for a few values of the relevant parameters. So far, the most efficient procedure for the data analysis comes from the concept of the photon transfer function, which is constructed by calculating the trajectories of a large number of photons (e.g., Laor 1991).

A further issue is effect of the relativistic transfer within the source. As pointed out by Cunningham (1976) the returning radiation may give an additional contribution to the illumination of the accretion disk. This effect was studied by Dabrowski et al. (1997) who found that the returning disk radiation in the Kerr metric gives no significant effect, in particular that it cannot enhance the equivalent width of the line by more than 20 $\%$. On the other hand, the light bending may become very important when one considers the transfer of the radiation from the X-ray source to the disk, leading to strong enhancement of the equivalent width of the iron line (Martocchia \& Matt 1996) and the amount of the Compton reflected radiation.

Figure 1 shows the emission line profiles from the accretion flows. The lines are typically broad and skewed rather than the double-peaked structures derived in the Newtonian approximation (cf. Fabian 1997 for the basic discussion of the effects in the line profiles). In general the characteristics of the lines allow to distinguish them from the line shapes produced by 


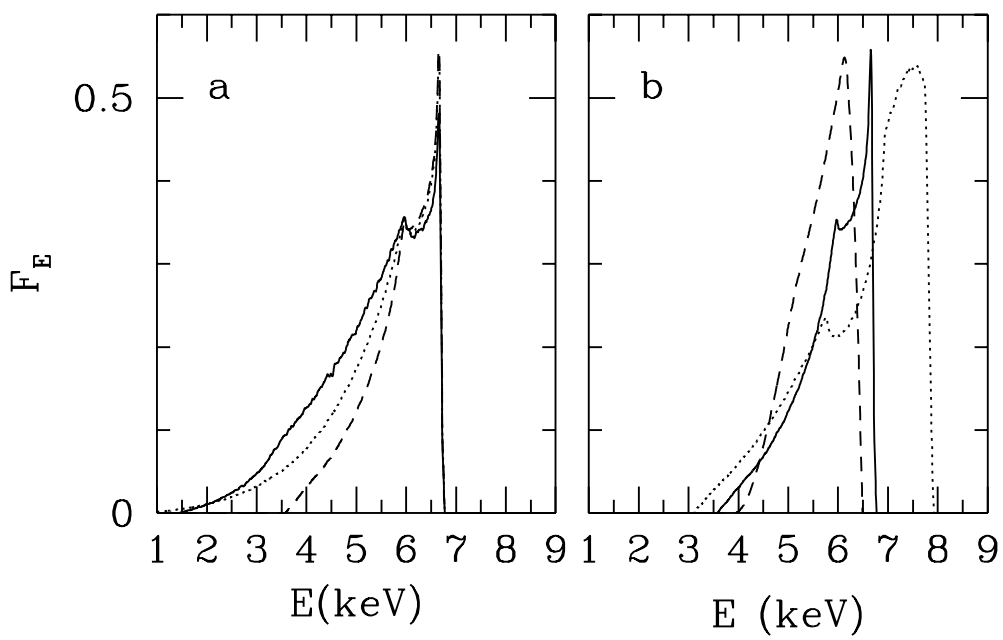

Figure 1. a) The dependence of the line profile on the geometry of the innermost part of the accretion flow. The rest energy of the line is assumed to be $6.4 \mathrm{keV}$ (neutral iron K $\alpha$ line). All the curves give the line profiles for the inclination angle $30^{\circ}$, the outer radius of the emission region, $r_{\text {out }}=100 r_{\mathrm{g}}$, and the radial emission law favoring the emission from the central parts (the local intensity of the emission of the line $I(r) \sim r^{-3}$ ). The dashed and dotted curves correspond to the Schwarzschild geometry with the inner radii, $r_{\text {in }}=6 r_{\mathrm{g}}$ and $2 r_{\mathrm{g}}$, respectively (the latter includes the contribution from the free-falling material). The solid curve corresponds to the Kerr geometry $(a=0.998)$ with $r_{\text {in }}=1.3 r_{\mathrm{g}}$. The Kerr metric disk gives more redshifted photons due to the location of the inner edge of the disk. Then, the high- $a$ line has an excess in the red tail, with respect to the low- $a$ line, if only the fluorescence from the circularly flowing disk material is taken into account. However, the contribution from the free-falling matter in the Schwarzschild metric removes this robust difference between the line profiles. b) The dependence of the line profile on the inclination angle. The curves correspond to the disk in the Schwarzshild metric extending between $6 r_{\mathrm{g}}$ and $100 r_{\mathrm{g}}$ with $I(r) \sim r^{-3}$, observed at $60^{\circ}$ (dotted curve), $30^{\circ}$ (solid curve) and $10^{\circ}$ (dashed curve). The position of the high energy peak of the line and the extent of the red tail can be used for the precise determination of the inclination and the distance of the emission region.

other physical processes (Fabian et al. 1995). The precisely measured line profile can be used for the determination of such system parameters as the inclination or the location of the emission region. However, the analysis of relativistic effects may likely lead to unreliable results, since modelling of the iron line and the iron edge is strongly dependent of the continuum model in most X-ray data (e.g., Zdziarski, Johnson \& Magdziarz 1996). Xray spectral deconvolution is a non-linear, complex procedure and it cannot be simplified by any indirect method of the analysis of the line profile. E.g., it is possible to constrain the parameters of the system using the extreme frequency shifts of the line profile (Bromley, Miller \& Pariev 1998), however, determination of these extreme shifts requires prior deconvolution of 

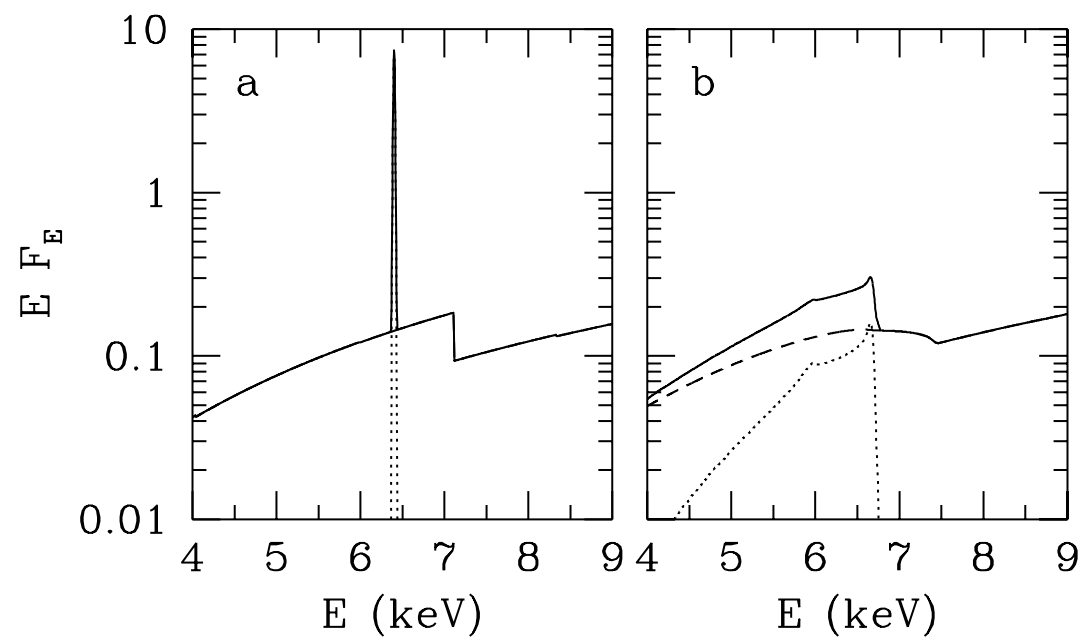

Figure 2. The figure compares the shape of the iron $\mathrm{K} \alpha$ line and absorption edge in the reflection spectrum arising from the X-ray irradiation of the slab at rest (a) with those in the reflection from the innermost region of the accretion disk in the Schwarzschild geometry (b) with $r_{\text {in }}=6 r_{\mathrm{g}}, r_{\text {out }}=100 r_{\mathrm{g}}, I(r) \sim r^{-3}$ and $\cos \theta_{\text {obs }}=30^{\circ}$. The solid curve shows the sum of the line (dotted curve; $E W=150 \mathrm{eV}$ in both geometries) and the Compton reflection (dashed curve). The relativistic transfer effects smear both spectral components, which add up giving rise to the complex spectral feature around $7 \mathrm{keV}$.

the line profile from the data, which yields by itself the best fitting set of the parameters of a given assumed model.

A particularly important, but usually neglected, effect is due to the relativistic distortion of the Compton reflected radiation (cf. Figure 2). The reflected continuum is affected similarly to the fluorescent line since it originates from the same matter (cf. Matt, Perola \& Piro 1991), although the dependence of reflection and fluorescence on ionization is different, and they are not necessarily smeared in the same manner. A further shortcoming of the usually applied models is the simplified treatment of the fluorescent line, which is approximated as a $\delta$-function. Due to Compton scattering of fluorescent photons in the disk, the line flux emerges locally as a broadened spectral feature, which effect is especially pronounced in highly ionized disks (e.g., Matt, Fabian \& Ross 1996). The above problems will be addressed in detail in our forthcoming paper (Maciołek-Niedźwiecki \& Magdziarz 1998). The spectral components, relevant to the determination of the iron line profile, are shown in the best fit of our model to the Ginga data of the Seyfert 1 galaxy MCG-6-30-15.

Finally, the innermost region of the accretion flow appears to be extremaly complex. It is well known, that solutions of the standard accretion 


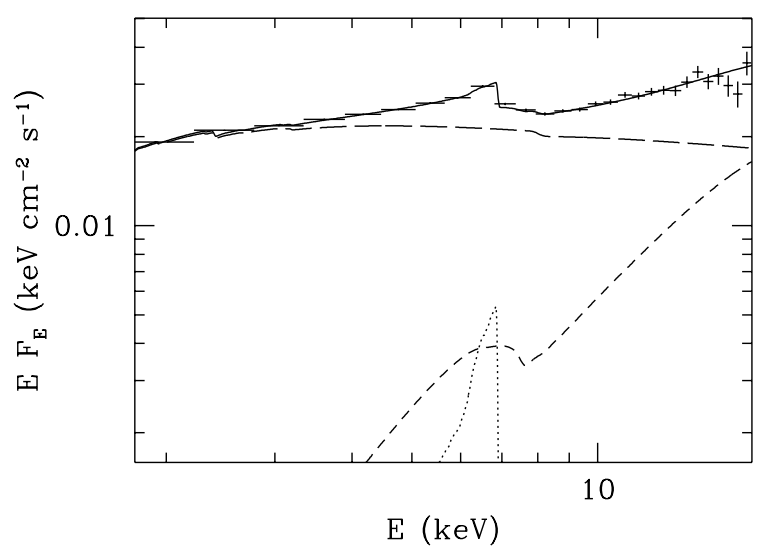

Figure 3. The average Ginga spectrum of MCG-6-30-15. The fitted model (solid curve) is a power-law (the energy spectral index $\alpha=1.1$ ), attenuated by the ionized absorber (long-dashed curve), the Compton reflection with the amplitude corresponding to the solid angle of the reprocessor as seen from the X-ray source of $3 \pi$ (for isotropic X-ray illumination; short dashed curve) and the Fe $\mathrm{K} \alpha$ line with $E W=300 \mathrm{eV}$ (dotted curve). All the components are self-consistently transferred through the Schwarzschild metric field with the assumption that the emission comes from the region between $6 r_{\mathrm{g}}$ and $100 r_{\mathrm{g}}$. The fitted radial emission law, $I(r) \sim r^{-3.9}$, and the inclination $\theta_{\mathrm{obs}}=40^{\circ}$. The fit weakly prefers the relativistically smeared spectrum over a reflection from a distant reprocessor. Furthermore, the distant reprocessor requires nearly edge-on inclination (in contradiction with its Seyfert 1 classification), as well as the solid angle subtended by the reflector $\sim 10 \pi$, which would require a complex model with strongly obscured X-ray source. On the other hand, the parameters of the relativistic model would be compatible with a nearly flat disk, provided that the isotropic X-ray source is close to the black hole horizon. In this case the light bending effects can account for both the very steep illumination of the disk (as implied by the fitted radial emissivity) and the enhanced amplitude of the iron line and Compton reflection.

disk with dissipation rate proportional to pressure are unstable (Shakura \& Sunyaev 1976) likely leading to the disk fragmentation (e.g., Krolik 1998). Such a complex structure of the central region has been indeed invoked to explain both the nature of EUV emission in AGNs in general (e.g., Kuncic, Celotti \& Rees 1997) and the overall spectral phenomenology in some particular sources (e.g., Magdziarz \& Blaes 1998; for NGC 5548). The complex radiative coupling of the multi-phase medium convolved with relativistic dynamics seems to be plausible alternative to a simple disk geometry. However, the profiles of the discrete features in such geometry need more complex treatment, and have not been calculated as yet. 


\section{Observational outline}

The presence of the broad iron lines, with profiles indicating strong relativistic effects, has been found in a number of Seyfert 1s observed by $A S C A$ (e.g., Nandra et al. 1997a). Such objects show no spectral contamination from jet activity and have relatively low absorption in the direction of the central part of the accretion flow. The iron line has typically $E W \sim 200 \mathrm{eV}$ and the profile consistent with that expected from an accretion disk with a bulk of emission coming from a region within $\sim 20 r_{\mathrm{g}}$. The observed peak energy at $6.4 \mathrm{keV}$ suggests that the emission comes mainly from nearly neutral matter, although the observed anticorrelation of the equivalent width of the broad line with the source luminosity (Nandra et al. 1997b) indicates that the reflecting matter tends to be more ionized in more luminous sources. It is also likely that the profiles contain a small $(\mathrm{EW} \sim 25 \mathrm{eV})$ constant and narrow component from a more distant reprocessor, e.g., a molecular torus. Orientation of the disk surface inferred for the sample of Seyfert 1 galaxies seems to be consistent with the unification scheme (Antonucci \& Miller 1985), however, an analysis of a sample of Seyfert 2s suggests that either a population of the obscured objects or geometry of the central part of the accretion flow is more complex (Turner et al. 1998; Elvis et al. 1998). We note, however, that the above results are likely to be subject to the modelling problems indicated in section 2 .

The most convincing example of relativistic broadening of the $\mathrm{Fe} \mathrm{K} \alpha$ line comes from observations of the Seyfert 1 galaxy MCG-6-30-15 (Tanaka et al. 1995; cf. Figure 4). The profile seems to vary with the source luminos-

ity in the sense that it shows stronger relativistic effects in fainter states of the source (Iwasawa et al. 1996). At the minimum state the profile seems to require either models with fluorescence from matter free-falling below the last stable orbit or models with maximally rotating black hole (cf. section 4). The signatures of the relativistic smearing of both the iron line and absorption edge have been also found in Ginga observations of Galactic black hole binaries: V404 Cyg (Życki, Done \& Smith 1997) and Nova Muscae (̇̇ycki, Done \& Smith 1998).

\section{The value of the black hole spin}

The spin of the black hole appears to be crucial for the properties of accretion powered objects, according to the unification scheme of AGNs, in which one expects that radio-loud jet dominated active galaxies host rapidly rotating black holes (cf. Blandford 1990). To test this unification model, one needs an independent measurement of the spin value. The mass of the black hole can be directly determined from the orbital motion in some X-ray binaries. A lower limit on $M$ can be derived for AGNs from the requirement that 


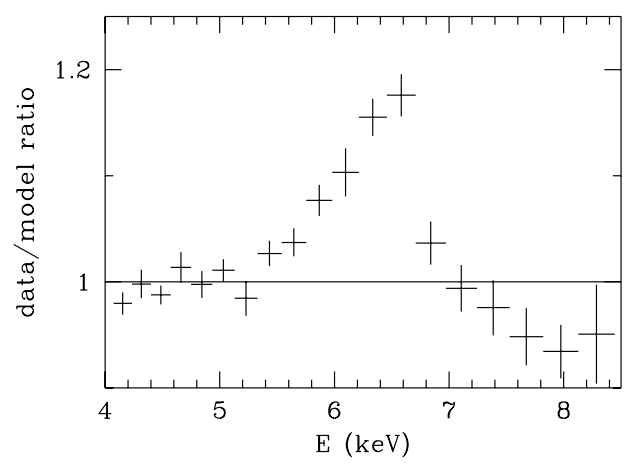

Figure 4. A ratio of the average data over power-law model for the ASCA observation of MCG-6-30-15 (July 1994). All four ASCA detectors where taken into account to achieve high signal-to-noise ratio which is sufficient to reveal the skewed profile of the line, with the maximum at $6.6 \mathrm{keV}$ and the broad tail at lower energies, independently of the assumed model of the continuum. The iron absorption edge above $7 \mathrm{keV}$ appears consistent with relativistic smearing.

their luminosity cannot exceed the corresponding Eddington limit. On the other hand, the estimation of the spin is based on more sophisticated arguments. The spectrum of the accretion disk depends on $a$ (Novikov \& Thorne 1973), however, we do not understand the reprocessing of the disk radiation sufficiently well to be able to constrain the spin value from the shape of the accretion disk spectrum, although the shape of the ultrasoft component in Galactic sources with superluminal jets may indicate that they are powered by rapidly rotating black holes (Zhang, Cui \& Chen 1997).

The effect of the black hole rotation on the line profile has been investigated, e.g., by Kojima (1991) who did not find any significant dependence of the line profile on $a$ if fluorescence occurs at a distance $r>6 r_{\mathrm{g}}$. As for the rapidly rotating black hole the disk extends well below $6 r_{\mathrm{g}}$, the emission from that region gives the extra flux in the red wing of the line, which makes the low- $a$ and high- $a$ lines clearly distinguishable (see Figure 1). Reynolds \& Begelman (1997) have pointed out, however, that fluorescence by the material inside the radius of marginal stability can have an observable influence on the iron line profile causing the red wing to be much wider. In fact, Jaroszyński (1997) finds that the difference in the velocity field, in the innermost region, between low- $a$ and high- $a$ cases does not yield any robust difference in the line profiles. Given the uncertainty in such parameters of the system as the radial emissivity law or inclination (which is not independently constrained, except for some black hole binaries, and must be found in the fitting procedure) we cannot expect to constrain the value of $a$ from the line profile with the present or even 
forthcoming X-ray data quality, unless we better understand the properties and geometry of the matter in the region inside $6 r_{\mathrm{g}}$. In fact, the model by Reynolds \& Begelman (1997) implies that the free-falling matter gets completely ionized and cannot contribute to the line profile, except for very inefficient $\mathrm{X}$-ray sources. The efficiency as low as $\eta \sim 10^{-5}$ is needed (where $L_{\mathrm{X}} \sim \eta \dot{m} c^{2}, L_{\mathrm{X}}$ is the $\mathrm{X}$-ray luminosity and $\dot{m}$ is the accretion rate) to avoid the complete ionization of that region. Such a low efficiency is very unlikely in the luminous accretion powered systems. Furthermore, the model by Reynolds \& Begelman (1997) relies on the central location of the X-ray source, which could be the case if the X-ray emission was related to the formation of the jet activity. However, if the emission is connected locally with the dissipation of the energy in the disk (as in the model with magnetic flares above the disk surface), one may expect that the line will be dominated by the flourescence occurring in the disk. There is virtually no dissipation of energy once the material has passed the last stable orbit, so in this case the contribution from the free-falling matter should not be very significant, independently of its ionization stage.

If any of the above arguments allows us to exclude the contribution from the free-falling matter, the observation of the very extended red tail of the line can be used as an unambiguous evidence for the rapid rotation of the central black hole.

\section{Summary}

$\mathrm{X}$-ray spectroscopy offers, for the first time, the unique opportunity to investigate the geometry of the region in the immediate vicinity of the black hole horizon. Currently available data suggest that the observed Xray spectra contain a significant contribution from matter emitting in the vicinity of the black hole, however, results are still uncertain mostly due to complex response of X-ray instruments, which confuses the iron line profile with both the iron edge and the underlying continuum. The progress in determination of the iron line and, consequently, the innermost geometry of the accretion flow, is expected to result from new X-ray observatories of high spectral resolution, to be launched in the near future.

Acknowledgements We are grateful to Greg Madejski for helpful discussion and his hospitality during our stay in NASA/GSFC where this paper has been written. We are also grateful to Chris Done for her comments and reviewing the manuscript.

\section{References}

Antonucci, R. R. \& Miller, J. S., 1985, ApJ, 297, 621

Bardeen, J. M., 1970, Nature, 226, 64 
Bardeen, J. M. \& Petterson, J. A., 1975, ApJ, 195, L65

Blaes, O. M., 1998, in: Accretion Processes in Astrophysical Systems: Some Like It Hot, eds. Holt, S. S., \& Kallman T. R.

Blandford, R. D., 1990, in: Active Galactic Nuclei, ed. J. L. Courvoisier, M. Mayor (Berlin: Springer)

Bromley, B. C., Miller, W. A. \& Pariev, V. I., 1998, Nature, 391, 54

Cunningham, C. T., 1975, ApJ, 202, 788

Cunningham, C. T., 1976, ApJ, 208, 534

Dabrowski, Y., Fabian, A. C., Iwasawa, K., Lasenby, A. N. \& Reynolds, C. S., 1997, MNRAS, 288, L11

Elvis, M., Fiore, F., Giommi, P. \& Padovani, P., 1998, ApJ, 492, 79

Fabian, A. C., 1997, in X-ray Imaging and Spectroscopy of Cosmic Hot Plasmas, ed. F. Makino, K. Mitsuda (Tokyo: Universal Academy Press)

Fabian, A. C., Rees, M. J., Stella, L. \& White, N. E., 1989, 238, 729

Fabian, A. C., et al., 1995, MNRAS, 277, L11

George, I. M. \& Fabain, A. C., 1991, MNRAS, 249, 352

Iwasawa, K., et al., 1996, MNRAS, 282, 1038

Jaroszyński, M., 1997, Acta Astronomica, 47,339

Jaroszyński, M. \& Kurpiewski, A., 1997, AA, 326, 419

Kojima, Y., 1991, MNRAS, 250, 629

Krolik, J. H., 1998, ApJL, in press

Kuncic, Z., Celotti, A. \& Rees, M. J., 1997, MNRAS, 284, 717

Laor, A., 1991, ApJ, 376, 90

Maciołek-Niedźwiecki, A. \& Magdziarz, P., 1998, in preparation

Madejski, G. M., 1998, in Theory of Black Hole Accretion Disks, eds. M. A. Abramowicz, G. Björnsson \& J. E. Pringle (Cambridge University Press), in press

Magdziarz, P. \& Blaes, O., 1998, in Proc. of IAU Symp. 188, Kyoto, Japan, in press

Magdziarz, P., Blaes, O., Zdziarski, A. A., Johnson, W. N. \& Smith, D. A., 1998, MNRAS, in press

Martocchia, A. \& Matt, G., 1996, MNRAS, 282, L53

Matt, G., Fabian, A. C. \& Ross, R. R., 1996, MNRAS, 278, 1111

Matt, G., Perola, G. C. \& Piro, L., 1991, AA, 247, 25

Moderski, R. \& Sikora, M., 1996, MNRAS, 283, 854

Nandra, K., George, I. M., Mushotzky, R. F., Turner, T. J. \& Yaqoob, T., 1997a, ApJ, 477,602

Nandra, K., George, I. M., Mushotzky, R. F., Turner, T. J. \& Yaqoob, T., 1997b, ApJ, 488, L91

Novikov, I. D. \& Thorne, K. S., 1973, in Black Holes, ed. C. DeWitt and B. DeWitt (New York: Gordon \& Breach)

van Paradijs, J., 1998, in The Many Faces of Neutron Stars, ed. R. Buccheri, J. van Paradijs, M. A. Alpar (Kluwer Academic Publishers), in press

Pounds, K. A., Nandra, K., Stewart, G. C., George, I. M. \& Fabian, A. C., 1990, Nature, 344,132

Poutanen, J., 1998, in Theory of Black Hole Accretion Disks, eds. M. A. Abramowicz, G. Björnsson \& J. E. Pringle (Cambridge University Press), in press

Reynolds, C. S. \& Begelman, M. C., 1997, MNRAS, 488, 109

Shakura, N. I. \& Sunyaev, R. A., 1976, MNRAS, 175, 613

Tanaka, Y., et al., 1995, Nature, 375, 659

Thorne, K. S., 1974, ApJ, 191, 507

Turner, T. J., George, I. M., Nandra, K. \& Mushotzky, R. F., 1998, ApJ, 493, 91

Zhang, S. N., Cui, Wei \& Chen, Wan, 1997, ApJ, 482, L155

Zdziarski, A. A., Johnson, W. N. \& Magdziarz, P.,1996, MNRAS, 283, 193

Życki, P. T., Done, C. \& Smith, D. A., 1997, ApJ, 488, L113

Życki, P. T., Done, C. \& Smith, D. A., 1998, ApJ, 496, L25 